\title{
Screening of Tomato Genotypes against Early Leaf Blight (Alternaria solani)
}

\author{
Misba Majeed ${ }^{1 *}$, Zaffar Afroz Badri ${ }^{1}$, Zahoor Ahmad Bhat ${ }^{1}$, M. Ashraf Bhat ${ }^{2}$, \\ M. A. Chattoo ${ }^{3}$, M.I. Makhdhoomi ${ }^{3}$ and Sajad Majeed Zargar ${ }^{4}$
}

${ }^{1}$ Division of Plant Pathology, SKUAST-Kashmir, ${ }^{2}$ Division of Genetics and Plant Breeding, SKUAST-Kashmir, ${ }^{3}$ Division of Vegetable Sciences, SKUAST-Kashmir, Division of Plant Biotechnology, SKUAST, Kashmir, India

*Corresponding author

\begin{tabular}{|l|}
\hline Ke y w o r d s \\
Tomato genotypes, \\
Alternaria solani, \\
Germplasms
\end{tabular}

\section{A B S T R A C T}

\begin{abstract}
An experiment was conducted for evaluation of tomato genotypes both in uninoculated field conditions and by artificial inoculated controlled conditions against early leaf blight at Research farm, SKUAST-Kashmir. A total of 50 tomato genotypes were screened under uninoculated field conditions. Among these germplasms, 07 test lines viz., 2014/TODHYB-7, 2015/TODHYB-2, 2016/TODHYB-4, 2016/TODHYB-6, 2016/TODHYB-7, 2016/TODVAR-10 and 2016/TODVAR-12 exhibited complete resistant against early blight and 14 lines viz., 2014/TODHYB-1, 2014/TODVAR-3, 2014/ TODVAR-1, 2014/TODVAR-4, 2014/TODVAR-6, 2015/TODVAR-1, 2016/TODVAR-3, 2016/ TODVAR-4, 2016/TODVAR-6, 2016/TODVAR-7, 2016/TODVAR-8, 2015/ TOINDVAR-2, 2015/TOINDVAR-3 and 2015/TOINDVAR-5 were found moderately resistant. The rest were found to exhibit moderately susceptible to susceptible reaction. The 21 genotypes found to be moderately resistant to resistant under field conditions were further screened under artificial inoculated controlled conditions. Three test lines (2016/TODHYB-4, 2016/TODHYB-7, 2016/TODHYB-6) were found resistant and six test lines (2016/TODVAR-10, 2016/TODVAR-3, 2015/TODHYB-2, 2014/TODHYB-7, 2016/TODVAR-6, 2016/TODVAR-12) were exhibited moderately resistant response. The rest were found to exhibit moderately susceptible to susceptible reaction.
\end{abstract}

\section{Introduction}

Tomato (Lycopersicon esculentum Mill.) is the second most important remunerable solanaceous vegetable crop after potato. It is native to South America and is widely cultivated in 140 countries of the world with an annual production of 150 million tons.
Varied climatic adaptability and high nutritive value has made tomato cultivation more popular in the recent years but the production has been fluctuating due to various diseases and insect pest damage. Tomato suffers from many diseases caused by fungi, bacteria, viruses, nematodes and abiotic factors (Balanchard, 1992). Among fungal diseases, 
early blight, caused by Alternaria solani is the most important disease in terms of reduction of yields and commercial values of production. The pathogen is not only responsible for damage to standing crop but also causing losses during transit and storage. The most efficient and economical method to manage plant diseases is the use of resistant varieties. Cultivation of resistant varieties can be effective approach to reduce the cost of cultivation, risk of development of resistance in pathogen, risk to human health and environmental pollution. In order to breed the varieties with durable resistance to the early blight of tomato, there is need for identification of source of resistance against the range of virulence present in the pathogen population. Although vast genetic diversity exists in well-adapted cultivars/germplasms in tomato in Kashmir, so far not much systematic study on resistance or susceptibility level of existing tomato genetic resources has been conducted. Therefore, the present study has been undertaken to screen the available genotypes against the pathogen.

\section{Materials and Methods}

A study was conducted to screen the tomato genotypes against early leaf blight both in uninoculated field conditions and artificial inoculated controlled conditions.

\section{Screening of genotypes under uninoculated field conditions}

Fifty tomato genotypes available with the Division of Vegetable Science, SKUAST-K, were screened under natural field conditions. The experiment was laid out at the Research Farm, Faculty of Agriculture, SKUAST-K. Plots of $2 \mathrm{~m} \times 2 \mathrm{~m}$ size were prepared and four weeks old seedlings were transplanted at recommended spacing of $60 \mathrm{~cm} \times 45 \mathrm{~cm}$. Recommended doses of fertilizers were applied. Disease intensity of early blight was recorded up to 90 days of transplanting to assess the level of resistance and susceptibility of each genotype using 0 - 5 rating scale of Datar and Mayee (1986). Following genotypes were screened (Table 1).

Screening of genotypes under artificially inoculated controlled conditions

Genotypes found resistant to moderately resistant under uninoculated field conditions were further screened under artificially inoculated controlled conditions Five-weekold seedlings of each genotypes were transplanted in the polyethylene bags containing sterilized soil. The seedlings were then inoculated by spraying conidial suspension of $A$. solani. Inoculated plants were covered with clear polyethylene cover for $72 \mathrm{~h}$ immediately after inoculation to allow infection (Bokshi et al., 2003). The disease intensity was recorded by visualizing the area of necrotic spots as per 0-5 scale given by Datar and Mayee (1986) (Table 2).

\section{Results and Discussion}

\section{Screening of genotypes under field conditions}

Fifty tomato genotypes which included 8 commercial cultivars and 42 germplasm lines were screened under field conditions against early blight disease. Out of the commercial cultivars, four (Shalimar-I, Shalimar-II, Arka Vikas and Kashi Anupama) showed moderately susceptible reaction with average disease intensity ranging from 27.83 to 38.23 per cent while as rest of cultivar lines (Swarna Ratna, Bhagya, BSS-488 and H-86) exhibited susceptible reaction with the average disease intensity ranging from 52.09 to 57.70 per cent respectively. Among 42 germplasm lines, fourteen (2014/TODHYB-1, 2014/TODVAR3, 2014/TODVAR-1, 2014/TODVAR-4, 2014/TODVAR-6, 2015/TODVAR-1 
2016/TODVAR-3, 2016/TODVAR-6, 2016/TODVAR-8, 2016/TODVAR-4, 2016/TODVAR-7, 2015/TOINDVAR-2, 2015/TOINDVAR-3, 2015/TOINDVAR-5) showed moderately resistant reaction with average disease intensity ranging from 10.11 to 24.69 per cent while as only seven (2014/TODHYB-7, 2015/TODHYB-2, 2016/TODHYB-4, 2016/TODHYB-7, 2016/TODHYB-6, 2016/TODVAR-10, 2016/TODVAR-12) exhibited resistant reaction with average disease intensity ranging from 4.06 to 8.79 per cent respectively (Table 3 ). None of the genotypes showed highly susceptible response. Thus, the evaluated genotypes were arbitrarily categorized into four groups on the basis of disease intensities recorded (Table 4).

Screening of genotypes under artificially inoculated controlled conditions

In order to validate the resistance, 21 genotypes found moderately resistant to resistant under uninoculated field conditionswere further screened against $A$. solani under artificially inoculated controlled conditions. Three test lines (2016/TODHYB4, 2016/TODHYB-7, 2016/TODHYB-6) were found resistant with average disease intensity ranging from 3.46 to 8.16 per cent, six test lines (2016/TODVAR-10, 2016/TODVAR-3, 2015/TODHYB-2, 2014/TODHYB-7, 2016/TODVAR-6, 2016/TODVAR-12) were exhibited moderately resistant response with average disease intensity ranging from 11.32 to 24.59 per cent, six test lines (2014/TODVAR-6, 2015/TODVAR-1, 2016/TODVAR-8, 2016/TODVAR-7, 2016/TODVAR-4, 2014/TODHYB-1) were found moderately susceptible with average disease intensity ranging from 29.86 to 42.74 per cent and other remaining six test lines (2015/TODINVAR-5, 2015/TODINVAR-2, 2015/TODINVAR-3, 2014/TODVAR-1, 2014/TODVAR-3, 2014/TODVAR-4) exhibited susceptible response with average disease ranging from 51.16 to 60.93 per cent respectively (Table 5).

Table.1 Screened Genotypes

\begin{tabular}{|l|l|l|l|l|l|}
\hline S. No & \multicolumn{1}{|c|}{ Genotype } & S. No & \multicolumn{1}{|c|}{ Genotype } & \multicolumn{1}{|c|}{ S. No } & \multicolumn{1}{c|}{ Genotype } \\
\hline $\mathbf{1}$ & Shalimar-I & 18 & 2015/TODHYB-3 & 35 & 2015/TODVAR-1 \\
\hline $\mathbf{2}$ & Shalimar-II & 19 & 2015/TODHYB-4 & 36 & 2016/TODVAR-1 \\
\hline $\mathbf{3}$ & Swarna Ratna & 20 & 2016/TODHYB-4 & 37 & 2016/TODVAR-3 \\
\hline $\mathbf{4}$ & Bhagya & 21 & 2016/TODHYB-6 & 38 & 2016/TODVAR-4 \\
\hline $\mathbf{5}$ & BSS-488 & 22 & 2016/TODHYB-7 & 39 & 2016/TODVAR-5 \\
\hline $\mathbf{6}$ & Arka Vikas & 23 & H-86 & 40 & 2016/TODVAR-6 \\
\hline $\mathbf{7}$ & Kashi Anupama & 24 & 2014/TODVAR-2 & 41 & 2016/TODVAR-7 \\
\hline $\mathbf{8}$ & 2014/TODHYB-1 & 25 & 2014/TODVAR-3 & 42 & 2016/TODVAR-8 \\
\hline $\mathbf{9}$ & 2014/TODHYB-2 & 26 & 2014/TODVAR-5 & 43 & 2016/TODVAR-10 \\
\hline $\mathbf{1 0}$ & 2014/TODHYB-3 & 27 & 2014/TODVAR-1 & 44 & 2016/TODVAR-12 \\
\hline $\mathbf{1 1}$ & 2014/TODHYB-4 & 28 & 2014/TODVAR-7 & 45 & 2015/TOINDVAR-1 \\
\hline $\mathbf{1 2}$ & 2014/TODHYB-5 & 29 & 2014/TODVAR-4 & 46 & 2015/TOINDVAR-2 \\
\hline $\mathbf{1 3}$ & 2014/TODHYB-6 & 30 & 2014/TODVAR-6 & 47 & 2015/TOINDVAR-3 \\
\hline $\mathbf{1 4}$ & 2014/TODHYB-7 & 31 & 2015/TODVAR-5 & 48 & 2015/TOINDVAR-5 \\
\hline $\mathbf{1 5}$ & 2014/TODHYB-8 & 32 & 2015/TODVAR-2 & 49 & 2015/TOINDVAR-6 \\
\hline $\mathbf{1 6}$ & 2015/TODHYB-1 & 33 & 2015/TODVAR-4 & 50 & 2015/TOINDVAR-8 \\
\hline $\mathbf{1 7}$ & 2015/TODHYB-2 & 34 & 2015/TODVAR-7 & & \\
\hline
\end{tabular}


Table.2 Disease intensity

\begin{tabular}{|l|l|}
\hline Reaction Category & Per cent Disease Index (PDI) \\
\hline Resistant & $0.0-9.0$ \\
\hline Moderately resistant & $10.0-25.9$ \\
\hline Moderately susceptible & $26.0-50.9$ \\
\hline Susceptible & $51.0-75.9$ \\
\hline Highly susceptible & $<76$ \\
\hline
\end{tabular}

Table.3 Screening of tomato genotypes against early leaf blight under uninoculated natural field conditions

\begin{tabular}{|c|c|c|c|}
\hline S. No. & Genotype & Disease intensity (\%) & Reaction \\
\hline 1 & Shalimar-I & 27.83 & MS \\
\hline 2 & Shalimar -II & 38.23 & MS \\
\hline 3 & Swarna Ratna & 57.70 & $\mathbf{S}$ \\
\hline 4 & Bhagya & 54.10 & $\mathbf{S}$ \\
\hline 5 & BSS-488 & 52.09 & $\mathbf{S}$ \\
\hline 6 & Arka Vikas & 30.66 & MS \\
\hline 7 & Kashi Anupama & 31.51 & MS \\
\hline 8 & 2014/TODHYB-1 & 19.56 & MR \\
\hline 9 & 2014/TODHYB-2 & 34.56 & MS \\
\hline 10 & 2014/TODHYB-3 & 40.35 & MS \\
\hline 11 & 2014/TODHYB-4 & 44.56 & MS \\
\hline 12 & 2014/TODHYB-5 & 54.56 & $\mathbf{S}$ \\
\hline 13 & 2014/TODHYB-6 & 29.12 & MS \\
\hline 14 & 2014/TODHYB-7 & 4.06 & $\mathbf{R}$ \\
\hline 15 & 2014/TODHYB-8 & 27.20 & MS \\
\hline 16 & 2015/TODHYB-1 & 28.70 & MS \\
\hline 17 & 2015/TODHYB-2 & 4.17 & $\mathbf{R}$ \\
\hline 18 & 2015/TODHYB-3 & 31.32 & MS \\
\hline 19 & 2015/TODHYB-4 & 35.68 & MS \\
\hline 20 & 2016/TODHYB-4 & 4.52 & $\mathbf{R}$ \\
\hline 21 & 2016/TODHYB-6 & 8.79 & $\mathbf{R}$ \\
\hline 22 & 2016/TODHYB-7 & 7.66 & $\mathbf{R}$ \\
\hline 23 & H-86 & 52.31 & $\mathbf{S}$ \\
\hline 24 & 2014/TODVAR-2 & 47.67 & MS \\
\hline 25 & 2014/TODVAR-3 & 20.69 & MR \\
\hline 26 & 2014/TODVAR-5 & 43.76 & MS \\
\hline 27 & 2014/TODVAR-1 & 24.69 & MR \\
\hline 28 & 2014/TODVAR-7 & 30.28 & MS \\
\hline 29 & 2014/TODVAR-4 & 15.28 & MR \\
\hline 30 & 2014/TODVAR-6 & 14.83 & MR \\
\hline 31 & 2015/TODVAR-5 & 54.71 & $\mathbf{S}$ \\
\hline 32 & 2015/TODVAR-2 & 51.28 & $\mathbf{S}$ \\
\hline 33 & 2015/TODVAR-4 & 54.86 & $\mathbf{S}$ \\
\hline
\end{tabular}




\begin{tabular}{|l|l|c|c|}
\hline $\mathbf{3 4}$ & $2015 /$ TODVAR-7 & 39.18 & MS \\
\hline $\mathbf{3 5}$ & $2015 /$ TODVAR-1 & 20.44 & MR \\
\hline $\mathbf{3 6}$ & $2016 /$ TODVAR-1 & 40.58 & MS \\
\hline $\mathbf{3 7}$ & $2016 /$ TODVAR-3 & 15.37 & MR \\
\hline $\mathbf{3 8}$ & $2016 /$ TODVAR-4 & 11.33 & MR \\
\hline $\mathbf{3 9}$ & $2016 /$ TODVAR-5 & 39.12 & MS \\
\hline $\mathbf{4 0}$ & $2016 /$ TODVAR-6 & 10.11 & MR \\
\hline $\mathbf{4 1}$ & 2016/TODVAR-7 & 13.48 & MR \\
\hline $\mathbf{4 2}$ & 2016/TODVAR-8 & 22.21 & MR \\
\hline $\mathbf{4 3}$ & 2016/TODVAR-10 & 4.91 & $\mathbf{R}$ \\
\hline $\mathbf{4 4}$ & 2016/TODVAR-12 & 7.29 & $\mathbf{R}$ \\
\hline $\mathbf{4 5}$ & 2015/TOINDVAR-1 & 48.45 & MS \\
\hline $\mathbf{4 6}$ & 2015/TOINDVAR-2 & 14.06 & MR \\
\hline $\mathbf{4 7}$ & 2015/TOINDVAR-3 & 16.54 & MR \\
\hline $\mathbf{4 8}$ & 2015/TOINDVAR-5 & 17.93 & $\mathbf{S}$ \\
\hline $\mathbf{4 9}$ & 2015/TOINDVAR-6 & 51.16 & $\mathbf{S}$ \\
\hline $\mathbf{5 0}$ & 2015/TOINDVAR-8 & 55.86 & \\
\hline
\end{tabular}

Table.4 Grouping of various tomato genotypes into different reactioncategories on the basis of their response to Early Blight disease

\begin{tabular}{|l|l|l|}
\hline Reaction category & $\begin{array}{l}\text { Percent disease } \\
\text { index (PDI) }\end{array}$ & Genotype \\
\hline Resistant & $\mathbf{0 . 0 - 9 . 0}$ & 2014/TODHYB-7, 2015/TODHYB-2, \\
& & 2016/TODHYB-4, 2016/TODHYB-6, \\
& & 2016/TODHYB-7, 2016/TODVAR-10, \\
2016/TODVAR-12
\end{tabular}


Table.5 Screening of genotypes under artificially inoculated controlled conditions

\begin{tabular}{|l|l|c|l|}
\hline S. No & Genotype & $\begin{array}{c}\text { Disease intensity } \\
\mathbf{( \% )}\end{array}$ & Reaction \\
\hline $\mathbf{1}$ & 2015/TODINVAR-5 & 51.16 & $\mathbf{S}$ \\
\hline $\mathbf{2}$ & 2015/TODINVAR-2 & 51.96 & $\mathbf{S}$ \\
\hline $\mathbf{3}$ & $2015 /$ TODINVAR-3 & 54.26 & $\mathbf{S}$ \\
\hline $\mathbf{4}$ & 2016/TODHYB-4 & 4.28 & $\mathbf{R}$ \\
\hline $\mathbf{5}$ & $2016 /$ TODVAR-10 & 13.86 & MR \\
\hline $\mathbf{6}$ & 2016/TODVAR-3 & 12.39 & $\mathbf{M R}$ \\
\hline $\mathbf{7}$ & 2014/TODVAR-6 & 42.74 & $\mathbf{M S}$ \\
\hline $\mathbf{8}$ & 2014/TODVAR-1 & 59.64 & $\mathbf{S}$ \\
\hline $\mathbf{9}$ & 2014/TODVAR-3 & 60.93 & $\mathbf{S}$ \\
\hline $\mathbf{1 0}$ & 2014/TODVAR-4 & 58.21 & $\mathbf{S}$ \\
\hline $\mathbf{1 1}$ & 2015/TODVAR-1 & 34.36 & $\mathbf{M S}$ \\
\hline $\mathbf{1 2}$ & 2016/TODHYB-7 & 3.46 & $\mathbf{R}$ \\
\hline $\mathbf{1 3}$ & 2016/TODVAR-8 & 34.41 & $\mathbf{M S}$ \\
\hline $\mathbf{1 4}$ & 2016/TODVAR-7 & 29.86 & $\mathbf{M S}$ \\
\hline $\mathbf{1 5}$ & 2015/TODHYB-2 & 13.07 & MR \\
\hline $\mathbf{1 6}$ & 2014/TODHYB-7 & 12.14 & MR \\
\hline $\mathbf{1 7}$ & 2016/TODVAR-4 & 30.83 & MS \\
\hline $\mathbf{1 8}$ & 2016/TODVAR-6 & 11.32 & MR \\
\hline $\mathbf{1 9}$ & 2016/TODHYB-6 & 8.16 & $\mathbf{R}$ \\
\hline $\mathbf{2 0}$ & 2016/TODVAR-12 & 24.59 & MR \\
\hline $\mathbf{2 1}$ & 2014/TODHYB-1 & 35.39 & MS \\
\hline
\end{tabular}

Table.6 Grouping of various tomato genotypes into different reaction categories on the basis of their response to early blight disease

\begin{tabular}{|l|l|l|}
\hline Reaction category & $\begin{array}{l}\text { Percent } \\
\text { disease index } \\
\text { (PDI) }\end{array}$ & Germplasm \\
\hline Resistant & $\mathbf{0 . 0 - 9 . 0}$ & $\begin{array}{l}\text { 2016/TODHYB-4, 2016/TODHYB-7, } \\
\text { 2016/TODHYB-6 }\end{array}$ \\
\hline Moderately resistant & $\mathbf{1 0 . 0 - 2 5 . 9}$ & $\begin{array}{l}\text { 2016/TODVAR-10, 2016/TODVAR-3, } \\
\text { 2015/TODHYB-2, 2014/TODHYB-7, } \\
\text { 2016/TODVAR-6, 2016/TODVAR-12 }\end{array}$ \\
\hline Moderately susceptible & $\mathbf{2 6 . 0 - 5 0 . 9}$ & 2014/TODVAR-6, 2015/TODVAR-1, \\
& & 2016/TODVAR-8, 2016/TODVAR-7, \\
\hline Susceptible & $\mathbf{5 1 . 0 - 7 5 . 9}$ & 2016/TODVAR-4, 2014/TODHYB-1 \\
\hline Highly susceptible & & 2015/TODINVAR-5, 2015/TODINVAR-2, \\
& & 2014/TODVAR-3, 2014/TODVAR-4 \\
\hline
\end{tabular}


Hence, the genotypes screened in artificially inoculated conditions were categorized into four different groups on the basis of disease intensities recorded (Table 6). None of the genotypes exhibited highly susceptible response.

Fifty tomato genotypes were screened under field conditions against early blight disease followed by screening of moderately resistant to resistant lines under artificially inoculated controlled conditions against single virulent isolate of $A$. solani. Among the test genotypes screened under field conditions, 7 were categorised as resistant, 14 as moderately resistant, 19 as moderately susceptible and remaining as susceptible. Of these 21 moderately resistant to resistant test lines, tested under controlled conditions only 3 were found resistant with resistant response against the test isolate, 6 were moderately resistant, another 6 were moderately susceptible and remaining all as susceptible. Similar efforts have been made earlier to identify the tomato genotypes with early blight resistance and tomato genotypes with varied levels of resistance have been identified by various workers (Upadhyay et al., 2009; Kumar and Srivastava, 2013; Kumar et al., 2015; Rani et al., 2015). Five Hybrids (2016/TODHYB-4, 2016/TODHYB-7, 2016/TODHYB-6, 2015/TODHYB-2, 2014/TODHYB-7) were observed to be resistant to moderately resistant among test lines claiming that the genetical factors from their parents would have contributed for the resistance in the hybrids. This finding is in agreement with Bharathkumar et al., (2017) who also reported that parental lines (IIHR1816, IIHR977, IIHR2850, IIHR2891 and IIHR2890) and some of their derived hybrids to be resistant or moderately resistant during screening of tomato genotypes against the disease. Thus, improvement programs from a base population with high genetic variability will increase the chances of establishing superior genotypes successfully in subsequent generations of selection (Hallauer and Miranda, 1988).

\section{References}

Balanchard, D. 1992. A colour atlas of tomato diseases. Wolfe Publishing Ltd., Brook House, London, p. 298.

Bharathkumar, M. V., Sadashiva, A. T., Anjanappa, M. and Amaranan jundeshwara. 2017. Screening a set of tomato parental lines and their hybrids for resistance to early blight (Alternaria solani) by detached leaf method. Journal of Applied and Natural Science 9 (3): $1305-1309$.

Bokshi, A., Morris, S. and Deverall, B. 2003. Effects of benzothiazole and acetylsalicylic acid on $\beta$-1, 3-glucanase activity and disease resistance in potato. Plant Pathology 52: 22-27.

Datar, V.V. and Mayee, C. D. 1986. Chemical management of early blight of tomato. Journal of Maharashtra Agriculture University3: 278-280.

Hallauer, A. R. and Miranda, F. J.B. 1988. Quantitative genetics in maize breeding. Iowa State University Press, Ames, $468 \mathrm{p}$

Kumar, S. and Srivastava, K. 2013. Screening of tomato genotypes against early blight (Alternaria solani) under field conditions. The Bioscan 8: 189-193.

Kumar, V., Naeem, F. and Kumar, A. 2015. Evaluation of Different Germplasms/ Cultivars of Tomato against Early Blight (Alternaria solani) in Field Conditions and by Artificial Inoculation Method: A Review Article. Open Access Library Journal, 2: 1-8.

Rani, S., Singh, R. and Gupta, S. 2017. Development of integrated disease management module for early blight of 
tomato in Jammu. Journal of Pharmacognosy and Phytochemistry, 6(2): 268-273.

Upadhyay, P., Singh, P.C., Sinha, B., Singh, M., Kumar, R., Pandey, K.K. and

Mathura, R. 2009. Sources of resistance against early blight (Alternaria solani) in tomato (Solanum lycopersicon). Indian Journal of Agricultural Sciences 79: 752-753.

\section{How to cite this article:}

Misba Majeed, Zaffar Afroz Badri, Zahoor Ahmad Bhat, M. Ashraf Bhat, M. A. Chattoo, M. I. Makhdhoomi and Sajad Majeed Zargar. 2020. Screening of Tomato Genotypes against Early Leaf Blight (Alternaria solani). Int.J.Curr.Microbiol.App.Sci. 9(01): 1140-1147. doi: https://doi.org/10.20546/ijcmas.2020.901.128 